Max-Planck-Institut für demografische Forschung

Max Planck Institute for Demographic Research

Konrad-Zuse-Strasse 1 - D-18057 Rostock - GERMANY

$\mathrm{Tel}+49$ (0) 3812081 - 0; Fax +49 (0) 3812081 - 202;

http://www.demogr.mpg.de

MPIDR WORKING PAPER WP 2009-043

DECEMBER 2009

\title{
How Ageing is shaped by Trade-offs
}

Annette Baudisch (baudisch@demogr.mpg.de)

(C) Copyright is held by the authors.

Working papers of the Max Planck Institute for Demographic Research receive only limited review. Views or opinions expressed in working papers are attributable to the authors and do not necessarily reflect those of the Institute. 


\section{How Ageing is shaped by Trade-offs}

by Annette Baudisch, 2009

The evolution of different life history strategies and thus different ageing patterns essentially depends on the nature of the underlying trade-offs between survival and reproduction. To fully comprehend ageing, we need to understand these trade-offs.

Keywords: ageing, senescence, trade-offs, survival, reproduction

\section{Ageing and Senescence}

"It is a curious thing that there is no word in the English language that stands for the mere increase of years: that is, for ageing silenced of its overtones of increasing deterioration and decay. ... We obviously need a word for mere ageing, and I propose to use 'ageing' itself for just that purpose. 'Ageing' hereafter stands for mere ageing, and has no other innuendo. I shall use the word 'senescence' to mean ageing accompanied by that decline of bodily faculties and sensibilities and energies which ageing colloquially entails. "This quotation, from the seminal paper on the evolution of senescence by Sir Peter Medawar in $1952^{1}$, highlights the deeply rooted association of ageing with deterioration. In this paper, I will use the terms 'ageing' vs. 'senescence' following Medawar's distinction.

The compound effects of age-specific change occurring both on the physiological and the genetic level are reflected in an organism's age-patterns of mortality and fertility. These patterns of ageing can be qualitatively different mortality and fertility could be (in various combinations) increasing, decreasing or constant (see ${ }^{2}$, Chapter 1 ). Thus, ageing can be associated with deterioration, maintenance or improvement with age. Senescence, in contrast, is only associated with patterns that correspond to deterioration and decay, i.e. increasing mortality and/or decreasing fertility over adult ages. One could speak of mortality-senescence or fertility-senescence each by itself. As a simple working definition, the term senescence is conventionally ${ }^{3}$ used to refer to mortality-senescence, a convention I will follow in this paper. 
Ageing, reflected in the age-patterns of mortality and fertility, is part of the life history strategy of a species. To the extent that the patterns of ageing are shaped by adaptive processes (for a discussion of evolutionary theories of ageing in general, and

of adaptive vs. non-adaptive processes shaping ageing in particular see ${ }^{2}$, Chapter 2 ), a species' pattern of ageing is determined by the species-specific trade-off between survival and reproduction. The more reproduction and the better survival, the higher is reproductive success. Those life history strategies that yield the highest lifetime reproductive success are favoured by Evolution. But a "Darwinian Demon” that exhibits immortality and infinite reproduction does not exist, because high reproduction and survival cannot be attained simultaneously - they compete for resources and entail direct and indirect costs to each other. Trade-offs are essential in studying ageing.

Discussing several theoretical models of the evolution of ageing and senescence, this working paper aims to demonstrate how different assumptions about the trade-off between survival and reproduction lead to qualitatively different conclusions about what ageing patterns are favoured by evolution. Some trade-offs are favourable for the evolution of senescence, other trade-offs are favourable for the evolution of sustenance (constant ageing patterns) or inverse senescence (improving ageing patterns).

\section{Trade-offs}

Survival and reproduction require resources and can imply detrimental effects to themselves and/or one another. For example, increasing reproduction at one age may cause damage that reduces or prevents survival and/or reproduction at later ages, e.g. the risk of damage in mating or in producing progeny ${ }^{4}$. Resources that drive survival and reproduction need to be acquired, which entails costs. Given the limited amount of acquired resources, they can either be invested in reproduction or survival. Higher survival often comes at the cost of lower reproduction. Resource acquisition trade-offs ${ }^{5}$ in the field of optimal forageing theory ${ }^{6-8}$, resource allocation trade-offs in life history theory ${ }^{9-13}$ (for a review see ${ }^{14}$, Section 5.3.4) and both allocation and acquisition trade-offs together ${ }^{15}$ have been extensively investigated. The models discussed in this paper mostly consider research allocation trade-offs. 
Based on the general trade-off between survival and reproduction, many life history models have been developed to understand how life histories are shaped by evolution, applying optimization models ${ }^{16,17}$. In these models, the evolutionary optimal balance of the trade-off between survival and reproduction yields those agepatterns of mortality and fertility that maximize lifetime reproductive success.

In applying optimization models in biology it is important to remember that "the concept of an optimum or fittest genotype may be deceptive, since the definition of an optimum is as ephemeral as the environment on which it is based,... it provides us with a powerful tool and is often the only useful model dealing with many biological problems", which is a quote from the seminal paper by Gadgil and Bossert $^{10}$. When I talk about an optimal life history strategy in this paper, I do it in the spirit of Maynard $\mathrm{Smith}^{18}$, who emphasized that " $[t]$ he role of optimization theories in biology is not to demonstrate that organisms optimize. Rather, they are an attempt to understand the diversity of life."

In the following discussion of life history optimization models, I will highlight what assumptions about the trade-off between survival and reproduction are responsible for producing qualitatively different patterns of ageing - in particular, what determines whether senescence, sustenance or inverse senescence evolve.

\section{Optimization Models of Ageing}

Kirkwood and Holliday Assuming specific functional forms, Kirkwood and Holliday ${ }^{19}$ (see also ${ }^{20}$ ) model mortality as increasing and fertility as decreasing exponential function with age depending on a shared parameter, which captures the overall decay of the organism. The larger this parameter, the faster is the decay in both survival and reproduction. Decay can be reduced by repair. The more the organism invests in maintenance, the slower is the decay in both survival and reproduction. Damage can thus be thought of as affecting the overall condition of the organism. The level of maintenance and thus decay is constant with adult age.

The trade-off between maintenance and reproduction is twofold: The higher the investment in maintenance, 1) the later reproduction starts, and 2) the lower is the initial, highest level of reproduction. Thus, the organism has to wait longer to get less profit per time unit, albeit over a longer period. The organism does not gain increased future reproductive potential by maintaining its physiological conditions but instead 
faces high costs in terms of late age of maturity and low levels of reproduction. With no prospects of increasing returns to investment in the future, such kind of trade-off favours the evolution of senescence.

The optimal level of maintenance, corresponding to a population growth rate of zero with all other levels corresponding to negative population growth, was estimated by fitting the model to mouse data. The parameters that fit these data correspond to an optimal strategy of senescence.

Abrams and Ludwig Different from the previous model, Abrams and Ludwig ${ }^{21}$ assume no particular functional forms for mortality or fertility. Instead, mortality is defined recursively. At every age, mortality increases by an amount of damage that accumulates. If at some age no damage occurs, mortality remains at its current level. Hence, age-specific mortality is assumed to be non-decreasing. The amount of damage accumulating at every age is assumed to be proportional to fertility, because more damage indicates that resources are diverted from survival to reproduction. Initial fertility at age zero equals zero. Since fertility is proportional to damage, age-specific fertility increases with the amount of damage accumulating at that age. The second derivative of fertility with respect to damage is negative. Though the functional form of fertility is not specified, it is assumed to be fixed, i.e. it is not subject to evolution in this model. The model allows reproduction to occur without damage to accumulate. This is an important feature of the trade-off because it allows parallel reproduction and maintenance of the organism.

The model is solved deriving the conditions for a maximum in reproductive value at every age with respect to the age-specific accumulation of damage. The endpoint condition depends on whether senescence or non-senescence is evolving. For the case of increasing mortality, the endpoint condition simply corresponds to maximize reproduction at the final age, which is defined as the age when mortality is so high that survival to the next age is negligible. Backward optimization finds the optimal strategy. In the case of non-senescence, mortality and fertility are maintained on a constant level. The dynamic programming equation can thus be solved since reproductive value is constant.

Abrams and Ludwig find that sustenance can be an optimal life history strategy. Given high age-specific survival, perfect repair is favoured if enough resources are available to ensure parallel reproduction and repair and if the gains in 
reproduction due to the marginal benefits of moving from a "no-damage" to a "damage" strategy are less then the costs of higher mortality. The authors note that this strategy may, however, not be a stable if mechanisms other than the resource allocation strategy could affect mortality. For instance, the non-adaptive process of mutation accumulation $^{1,22}$ could lower age-specific survival down to a point where the high-survival conditions would no longer be met and maintenance would become a suboptimal strategy. In case of senescent strategies, numerical solutions are derived to determine what kind of patterns of fertility in this model imply what optimal patterns of mortality, thereby exploring the nature of the trade-off between reproduction and repair. Alternative model versions are developed.

The models by Abrams and Ludwigs make a strong case for how alternative assumptions about the trade-off between reproduction and repair can lead to alternative patterns of mortality, including maintenance of the organism. Low risk of death and the option of efficient parallel repair and reproduction promote nonsenescent strategies. Senescent strategies of various shapes are found when reproduction is costly in terms of accumulating damage.

The range of possible ageing patterns resulting from the Abrams-Ludwig models is wider than in the Kirkwood-Holliday model, because the functional forms of mortality and fertility in the condition for the optimal strategy are not specified. In particular, sustenant ageing patterns are not a priory excluded - the model allows for parallel maintenance (i.e. constant mortality) and non-zero reproduction - and indeed sustenance, in this model, does evolve. Note that the model assumes a direct trade-off between mortality and fertility mediated via a common 'damage’ parameter.

Kozlowski and Cichon Kozlowski and Cichon ${ }^{23-26}$ develop models that optimize resource allocation assuming trade-offs between growth in size, repair of somatic damage and reproduction. As in the Abrams-Ludwig model, mortality is defined recursively being a non-decreasing function of age. At every age, damage accumulates depending on the amount of repair. Repair-effects are modelled nonlinearly. Different to the Abrams-Ludwig model, zero damage accumulation can only be achieved by investing all available energy in repair. Thus, zero damage comes at the costs of zero reproduction. Parallel reproduction and maintenance is thereby excluded, i.e. this specification of the trade-off implies that a non-senescent strategy cannot be evolutionary optimal. 
In an extended version of this model, Cichon and Kozlowski ${ }^{25}$ assume that mortality not only depends on the accumulation of damage, but also on size ${ }^{\mathrm{a}}$. As size increases, mortality falls. Growth in size is costly; it competes for resources with repair and reproduction. The trade-off between growth and reproduction is modelled in a linear way, which yields optimal solutions of exclusive growth or reproduction. Therefore this model captures species that stop growing when reproductive life starts. As in their initial models, full repair can only be achieved at the cost of zero reproduction. Optimal strategies therefore correspond to patterns of senescence.

The model is solved by backward programming, maximizing reproductive value at every age. The endpoint condition is assumed to correspond to the age when the probability of surviving from birth to that age is less than one in a thousand. This maximum lifespan is an outcome of the model. Note that this specification of maximum lifespan implicitly assumes that remaining reproduction after this age, i.e. when less then one in a thousand survive, is negligible. Many trees and fish produce millions of seeds or eggs, and thousands of saplings and baby fish, but only few, i.e. very much less then one in a thousand, survive to large sizes at which reproduction is plentiful. However, since such strategies of parallel growth and reproduction are not favoured by the linear trade-off between reproduction and growth assumed in the model, this should no be a problem here.

Note that for any optimal life history model of the type discussed in this paper it can be concluded: If there is an age after which future gains in reproduction are zero, then at this age it can never be optimal to invest in maintenance. Instead, it should always be optimal to invest everything that is left into reproduction. Thus the existence of a final age of reproduction makes senescence an inevitable outcome of the model, at least senescence over ages close to this final age. Abrams and Ludwig get around this problem by distinguishing between two possible cases: maintenance vs. senescence. In the maintenance case, the endpoint is simply given by the constant state of the organism reflected in constant birth and death rates and thus a constant reproductive value. In the senescence case, there is no problem assuming a finite age

\footnotetext{
a Note that the damage-dependent part of the mortality function is referred to as "senescence" and the size-dependent part is interpreted as capturing "extrinsic mortality". Williams et al. (2006) ${ }^{27}$ discuss various conceptual and methodological issues regarding definitions and measurements in studies of senescence.
} 
because if age-specific mortality increases, there clearly exists an age when survival to the next age becomes negligible and thus reproductive prospects are zero.

The models by Kozlowski and Cichon find that a variety of life history strategies can be optimal ranging from fast to slow senescent, from short to long lifespan. All strategies, as implied by the trade-offs assumed, correspond to patterns of determinate growth and increasing mortality across adult life. Senescence is the only possible ageing pattern that results from this model, since mortality is assumed to be a non-decreasing function and constant mortality can only be achieved at the cost of zero reproduction - mortality must increase. Note another factor that makes investment in future reproduction and survival sub-optimal: reproduction is constrained from increasing significantly in the future. Since reproduction is assumed to be proportional to an organism's size and since the linear trade-off between growth and reproduction implies that optimal strategies correspond to trajectories of exclusive growth and reproduction - growth ceases when reproduction begins - the size at reproductive maturity sets a limit to future reproductive potential.

Mangel and Munch ${ }^{28,}$, Initially developed to predict the evolution of compensatory growth ${ }^{28}$, Munch and Mangel $^{29}$ present an optimization model to explain mortality patterns at pre-reproductive ages. Different to the models described above, the trade-off between mortality and fertility is not direct, but is instead mediated via physiological variables - size and damage. They describe the state of an organism at every age. Mortality is inversely related to size and proportional to the level of damage. The change in size and damage over time is regulated by to the amount of foraging activity which is to be optimized. Mortality from predation increases with increasing foraging activity. The trade-offs in this model thus include energy acquisition costs.

More activity leads to higher energy intake that increases the total amount of energy available for growth and the repair of damage, but more activity also increases the risk of predation and the level of metabolism. Metabolism causes damage and requires energy. Growth increases size. A larger size lowers the risk of death and raises the ability to take in energy intake, but being big requires more energy for repair and higher levels of metabolism. Most trade-offs are nonlinear, the functional forms assumed are motivated by the mass scaling of West, Brown and Enquist ${ }^{30}$. 
Parameters have mechanistic interpretations and most of them are theoretically measurable.

The model is solved backwards via a dynamic programming equation seeking the level of activity that maximized reproductive value at every age. The endpoint is assumed to be the onset of reproduction. Residual reproductive value at maturity is assumed to be proportional to size and inversely related to damage. The model is analyzed across a wide range of parameters adopting a Monte Carlo approach.

Though not studying patterns over adult ages, i.e. ages when senescence could occur, this model provides insights into the evolution of ageing across juvenile ages. The model results show that mortality patterns across juvenile ages can be diverse, ranging from declining to broadly u-shaped trajectories. This diversity of results is enabled by the flexible nature of the assumed trade-offs. Trade-offs link reproduction and survival via physiological variables, are nonlinear and do not a priori constrain mortality or fertility to be increasing or decreasing functions with age. The model by Mangel and Munch highlights the power of a state-based approach in modeling mortality and fertility.

Vaupel, Baudisch and colleagues ${ }^{2,31}$ Similar to the model by Mangel and Munch, Vaupel and colleagues assume that mortality, fertility and growth are determined by an organism's physiological state. A “larger” state implies lower mortality and more resources available for maintenance, growth and reproduction, but also higher costs of maintenance. The change in state is driven by the balance between damage and repair. Physiological state can improve, deteriorate or remain the same over adult ages, depending on the allocation of resources between growth and reproduction. Damage occurs proportionally to the level of the state variable. Damage that occurs can be repaired. Thus, damage accumulation in this model is not inevitable.

We developed different models by alternative specification of the state variable. The simplest, first model is solved applying optimal control theory, the other models are solved using dynamic programming as done in the models discussed above: the optimal patterns of mortality and fertility result from the optimal resource allocation schedule over the course of life, which maximizes lifetime reproductive success. Different to the previous models, the endpoint condition is state specific. Energy in the final state is equal to zero: mortality is therefore infinite and remaining 
reproduction is zero. The optimal strategy is found following a backward procedure from final to initial state. At every level of state the fraction of energy allocated to repair (as opposed to reproduction) is optimized.

The models find that age-patterns of mortality and fertility can be diverse. They can go up, down, or remain constant over adult ages. Thus besides senescence, sustenance and inverse senescence - life histories of maintenance and improvement can be optimal.

The models by Vaupel, Baudisch and colleagues are the first that are capable to embrace the full scope of ageing patterns over adult ages. Whether or not senescence is the optimal strategy crucially depends on the shape of the trade-off between reproduction and maintenance. In our vitality model ${ }^{2}$, concave trade-offs lead to inverse senescence, linear trade-offs lead to sustenance and convex trade-offs lead to senescence. These results strongly motivate future investigation of the shape of the survival-reproduction trade-offs.

\section{Conclusion}

The models presented in this paper demonstrate how model assumption about the trade-off between survival and reproduction determine the range of predicted possible ageing patterns. Crucial points in the model assumptions are linearity vs. non-linearity in the trade-offs, inclusion or exclusion of mediating variables that determine either or both mortality and fertility, endpoint conditions of the problem's time-horizon, future returns to current investment reflected in the potential for indeterminate growth, and constraints on the qualitative shape of mortality and fertility patterns.

The aim of this paper was to highlight the important role of trade-offs in shaping ageing, using several optimization models of ageing as examples. Other models not discussed here have been developed to study the evolution of ageing (e.g. ${ }^{32-35}$ ) that incorporate different variables and processes like intergenerational transfers $^{32,33}$ or density dependence ${ }^{34}$. Processes like intra- and inter-species resource transfers, density dependent population regulation, sexual selection, competition, environmental variability, migration - the list goes on - affect ageing. Species differ with respect to uncountable characteristics, and we do not yet understand what characteristics are essential to include in models of ageing for different kinds of 
organisms. Studying different models of ageing for different species including different types of trade-offs will enhance understanding of the evolution of ageing across the tree of life.

\section{Acknowledgement}

I am grateful for many insightful discussions with and feedback from James W. Vaupel, Linda Partridge, and Jessica Metcalf.

\section{References}

1. Medawar, P.B. An Unsolved Problem of Biology, in Uniqueness of the Individual 44-70 (H.K. Lewis, London; 1952).

2. Baudisch, A. Inevitable Senescence? Contributions to evolutionarydemographic theory. (Springer, Berlin, Heidelberg; 2008).

3. Finch, C.E. Longevity, senescence, and the genome. (1990).

4. Sgro, C.M. \& Partridge, L. A delayed wave of death from reproduction in Drosophila. Science 286, 2521-2524 (1999).

5. Van Noordwijk, A.J. \& De Jong, G. Acquisition and allocation of resources their influence on variation in life history tactics. American Naturalist 128, 137-142 (1986).

6. Charnov, E.L. Optimal foraging, marginal value theorem. Theoretical Population Biology 9, 129-136 (1976).

7. Pyke, G.H., Pulliam, H.R. \& Charnov, E.L. Optimal foraging - selective review of theory and tests. Quarterly Review of Biology 52, 137-154 (1977).

8. $\quad$ Stephens, D.W., Krebs, J.R., Stephens, D.W. \& Krebs, J.R. Foraging theory. Foraging theory., i-xiv, 1-247 (1986).

9. Charlesworth, B. \& Leon, J.A. Relation of reproductive effort to age. American Naturalist 110, 449-459 (1976).

10. Gadgil, M. \& Bossert, W.H. Life Historical Consequences of Natural Selection. The American Naturalist 104, 1-24 (1970).

11. Leon, J.A. Life histories as adaptive strategies. Journal of Theoretical Biology 60, 301-335 (1976).

12. Pianka, E.R. \& Parker, W.S. Age-specific reproductive tactics. American Naturalist 109, 453-464 (1975). 
13. Schaffer, W.M. Selection for optimal life histories: The effects of age structure. Ecology 55, 291-303 (1974).

14. Charlesworth, B. Evolution in age structured populations. Cambridge Studies in Mathematical Biology 13, i-xiii, 1-306 (1994).

15. De Jong, G. \& Van Noordwijk, A.J. Acquisition and allocation of resources genetic covariances selection and life histories. American Naturalist 139, 749770 (1992).

16. Roff, D.A. Life history evolution. (2002).

17. Stearns, S.C. The evolution of life histories. (1992).

18. Smith, J.M. Optimization theory in evolution. Annual Review of Ecology and Systematics 9, 31-56 (1978).

19. Kirkwood, T.B.L. \& Holliday, R. Aging as a consequence of natural selection. Bittles, a. H. and K. J. Collins (Ed.). Symposia of the Society for the Study of Human Biology, 25. the Biology of Human Ageing; Joint Symposium of the Society for the Study of Human Biology and the British Society for Research in Ageing, London, England, Apr. 1984. Viii+280p. Cambridge University Press: Cambridge, England; New York, N.Y., USA. Illus, 1-16 (1986).

20. Kirkwood, T.B.L. \& Rose, M.R. Evolution of senescence - late survival sacrificed for reproduction. Philosophical Transactions of the Royal Society of London Series B-Biological Sciences 332, 15-24 (1991).

21. Abrams, P.A. \& Ludwig, D. Optimality theory, Gompertz' law, and the disposable soma theory of senescence. Evolution 49, 1055-1066 (1995).

22. Hamilton, W.D. Moulding of senescence by natural selection. Journal of Theoretical Biology 12, 12-\& (1966).

23. Cichon, M. Evolution of longevity through optimal resource allocation. Proceedings of the Royal Society of London Series B-Biological Sciences 264, 1383-1388 (1997).

24. Cichon, M. Diversity of age-specific reproductive rates may result from ageing and optimal resource allocation. Journal of Evolutionary Biology 14, 180-185 (2001).

25. Cichon, M. \& Kozlowski, J. Ageing and typical survivorship curves result from optimal resource allocation. Evolutionary Ecology Research 2, 857-870 (2000). 
26. Kozlowski, J. Optimal Allocation of Resources Explains Interspecific LifeHistory Patterns in Animals with Indeterminate Growth. Proceedings: Biological Sciences 263, 559-566 (1996).

27. Williams, P.D., Day, T., Fletcher, Q. \& Rowe, L. The shaping of senescence in the wild. Trends in Ecology \& Evolution 21, 458-463 (2006).

28. Mangel, M. \& Munch, S.B. A life-history perspective on short- and long-term consequences of compensatory growth. American Naturalist 166, E155-E176 (2005).

29. Munch, S.B. \& Mangel, M. Evaluation of mortality trajectories in evolutionary biodemography. Proceedings of the National Academy of Sciences of the United States of America 103, 16604-16607 (2006).

30. West, G.B., Brown, J.H. \& Enquist, B.J. A general model for ontogenetic growth. Nature 413, 628-631 (2001).

31. Vaupel, J.W., Baudisch, A., Dolling, M., Roach, D.A. \& Gampe, J. The case for negative senescence. Theoretical Population Biology 65, 339-351 (2004).

32. Chu, C.Y.C. \& Lee, R.D. The co-evolution of intergenerational transfers and longevity: An optimal life-history apporach. Theoretical Population Biology 69, 193-201 (2006).

33. Robson, A.J. \& Kaplan, H.S. Why do we die? American Economic Review 97, 492-495 (2007).

34. Seymour, R.M. \& Doncaster, C.P. Density dependence triggers runaway selection of reduced senescence. PLoS Computational Biology 3, e256 (2007).

35. Sozou, P.D. \& Seymour, R.M. To age or not to age. Proceedings of the Royal Society B-Biological Sciences 271, 457-463 (2004). 